\title{
Economic evaluation of a patient and carer centred system of longer-term stroke care from a cluster randomised trial (the LoTS care trial)
}

\author{
Anita Patel ${ }^{1 *}$, Anne Forster ${ }^{2,3}$, John Young ${ }^{3}$, Jane Nixon ${ }^{2}$, Katie Chapman $^{3}$, Martin Knapp ${ }^{4}$, Kirste Mellish ${ }^{3}$, \\ Ivana Holloway ${ }^{2}$, Amanda Farrin ${ }^{2}$
}

From Health Services Research: Evidence-based practice

London, UK. 1-3 July 2014

\section{Background}

Stroke generates considerable personal and financial burdens to society. We evaluated the cost-effectiveness of a new post-discharge system of care for stroke care co-ordinators (SCCs) to address the longer term problems experienced by stroke patients and their carers.

\section{Materials and methods}

A pragmatic cluster, randomised, controlled trial compared the system of care against usual care. Randomisation was at the level of stroke service. Participants' use of health/social care services and informal care were measured by self-complete questionnaires at baseline, 6 and 12 months. From these, we estimated and compared individual-level total costs from health/social care and societal perspectives at 6 months, 12 months and over 1 year. Costs were combined with the primary outcome, psychological health (General Health Questionnaire 12; GHQ12), and quality-adjusted life years (QALYs; based on the EQ-5D) to examine cost-effectiveness at 6 months. Costeffectiveness acceptability curves based on the net benefit approach and bootstrapping techniques were used to estimate the probability of cost-effectiveness.

\section{Results}

32 services were randomised, of which 29 participated, and 800 stroke patients (401 intervention, 399 control) and 208 carers (108 intervention, 100 control) were recruited. Costs of SCC inputs (mean difference $£ 42$; $95 \%$ CI: $-30,116)$ and total health and social care costs at 6 months, 12 months and over 1 year were similar between groups. Total costs from the societal perspective were higher in the intervention group due to greater use of informal care $(+£ 1163$ at 6 months, 95\% CI 56 to 3271; $+£ 4135$ at 12 months, $95 \%$ CI 618 to 7652 ). There were no differences in GHQ12 or QALYs and the probability of the system of care being cost-effective at 6 months was low at the current policy threshold of $£ 20,000$ to $£ 30,000$ per QALY gain.

\section{Conclusions}

The system of care was not cost-effective compared with usual care in this patient group over the period we examined. It is unclear why the intervention group accessed greater levels of informal care.

\section{Authors' details}

'Institute of Psychiatry, King's College London, London, UK. ${ }^{2}$ Faculty of Medicine \& Health, University of Leeds, Leeds, UK. ${ }^{3}$ Academic Unit of Elderly Care and Rehabilitation, Bradford Teaching Hospitals NHS Foundation Trust Bradford, UK. ${ }^{4}$ Personal Social Services Research Unit, London School of Economics \& Political Science, London, UK.

Published: 7 July 2014

doi:10.1186/1472-6963-14-S2-P92

Cite this article as: Patel et al: Economic evaluation of a patient and carer centred system of longer-term stroke care from a cluster randomised trial (the LoTS care trial). BMC Health Services Research 2014 14(Suppl 2):P92. 